\title{
A SIMPLIFIED SMAHTR BENCHMARK PROBLEM SET
}

\author{
K. Lisa Reed ${ }^{1}$, Farzad Rahnema ${ }^{1}$ \\ ${ }^{1}$ Georgia Institute of Technology \\ North Ave NW, Atlanta, GA \\ kreed34@gatech.edu, farzad@gatech.edu
}

\begin{abstract}
The Small, Modular Advanced High Temperature Reactor (SmAHTR) is a preconceptual design for a fluoride salt-cooled small modular reactor (SMR) [1]. In this paper, a stylized 2D benchmark problem set has been created based on SmAHTR. Certain gaps and considerations in burnable poison and control rod content were unspecified/undetermined in the preconceptual design, but those gaps were filled for the stylized problem set. With those features, this problem set could then be used for benchmarking neutron transport methods as well as its low order methods in 2D single assembly and full core configurations.

For this benchmark set, continuous energy Monte Carlo calculations were performed. Those calculations provided $k_{\text {eff }}$ values of $0.9459( \pm 11 \mathrm{pcm})$ and $1.1436( \pm 12 \mathrm{pcm})$ in the full core configuration with all the control rods fully inserted and withdrawn, respectively. The single assembly calculations yielded an eigenvalue, $\mathrm{k}_{\text {inf }}$ of $0.9987( \pm 15 \mathrm{pcm})$ and $1.2117( \pm 15 \mathrm{pcm})$ with all of the control rods either inserted or removed, respectively. In the full core configuration, the worth of all the control rods and burnable poison particles were determined to be $197.6( \pm 0.16) \mathrm{mk}$ and $311.6( \pm 0.23) \mathrm{mk}$, respectively. The corresponding results in the single assembly configurations are $213( \pm 0.21) \mathrm{mk}$ and $337.4( \pm 0.20) \mathrm{mk}$, respectively. A near-critical configuration was also determined for the reactor by inserting control rods in some assemblies, thus providing a case with a $\mathrm{k}_{\text {eff }}$ value of $0.9909( \pm 12 \mathrm{pcm})$.
\end{abstract}

KEYWORDS: FHR, MSR, SMR, Benchmark

\section{INTRODUCTION}

The Oak Ridge National Laboratory report on the preconceptual SmAHTR discusses parameters and specifications essential to the design, such as the fuel type, coolant, and core configuration. The fuel choices discussed in the report are different forms of graphite-suspended TRISO particles including plank fuel type, annular pin fuel type, and cylindrical pin fuel type. Although all of these options for assembly configuration were discussed in the paper, the cylindrical pin fuel type is of consideration for this set of stylized benchmark problems. This design however did not specify any control mechanisms for the core in either the form of burnable poison or control rods. To create a complete set of benchmark problems containing controlled and critical cases, the addition of control mechanisms was necessary and discussed below.

\section{DESIGN PARAMETERS AND SIMPLIFICATIONS}


The geometry and composition of the core as well as the simplifications regarding them are discussed in this section. While much of the core design was specified, certain simplifications were necessary in the creation of the 2D benchmark problem set, and gaps in reactivity control needed to be addressed.

\subsection{Assembly and Core Geometry}

During the development of this problem set, related work regarding the thermal hydraulic model for the design was simultaneously performed, and thermal hydraulic considerations were adapted into this neutronics benchmark as important modifications from a neutronics standpoint were likewise adapted into the thermal hydraulics model [2]. Within the assembly, several modifications were made to reduce the amount of bypass flow in the reactor, helping limit coolant temperature to the desired bounds of the original preconceptual design. The shape of the coolant channel in the assembly was changed from a cylinder of $16.94 \mathrm{~cm}$ radius to a hexagonal channel with a $16.132 \mathrm{~cm}$ apothem to reduce the bypass in the large areas between the outer walls of the channels and the pins as well as preserve the flow area of the assembly. In addition, to reduce the distance between the outer row of pins and the wall of the assembly coolant channel, the pin pitch was increased from $3.08 \mathrm{~cm}$ to $3.29 \mathrm{~cm}$ to redistribute the pins and create more even gap spacing between pins and the walls. While this significantly affects the thermal hydraulic properties of the reactor and reduces bypass flow, neutronic properties are largely unaffected by this change, as the ratio of fuel pin area to molten salt flow area to graphite reflector is preserved. Pictured below is the assembly before and after the modifications where fuel pin locations are represented in green and graphite pins in grey. Control rod locations and burnable poison pin locations are specified in yellow and blue in the new design, respectively, and the addition of these pins will be discussed later in section 2.3.
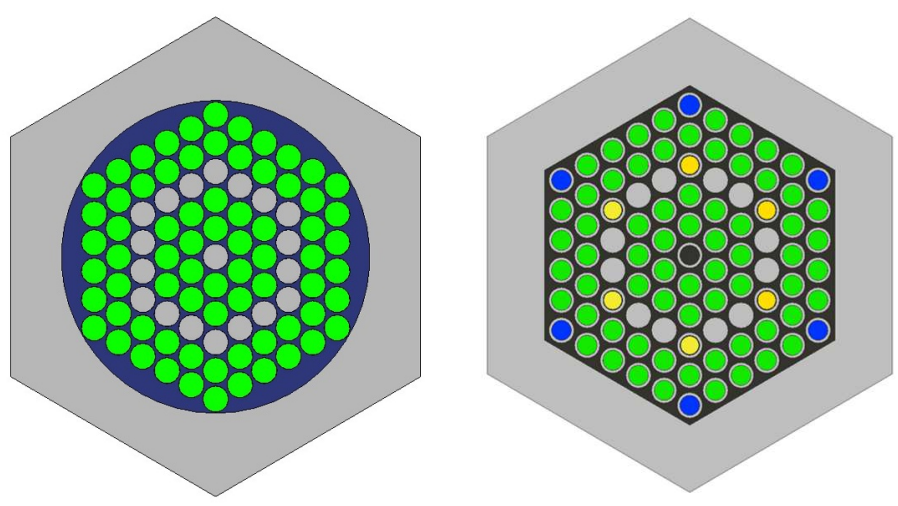

Figure 1. Original Assembly (Left) and Modified Assembly (Right).

The core itself comprises 19 of these assemblies which in 3D, span a height of 4 meters and span a diameter of 3 meters with the graphite reflector. The graphite reflector is cylindrical shaped with an outer radius of 1.5 meters that encompass all space not occupied by the assemblies. Within the reflector, $6 \mathrm{~cm}$ radius coolant channels are placed with a $45 \mathrm{~cm}$ pitch from the center of the outer row of assemblies in the same hexagonal array the assemblies form. Figure 2 below shows a rendering of the reactor core cross section with those channels. 


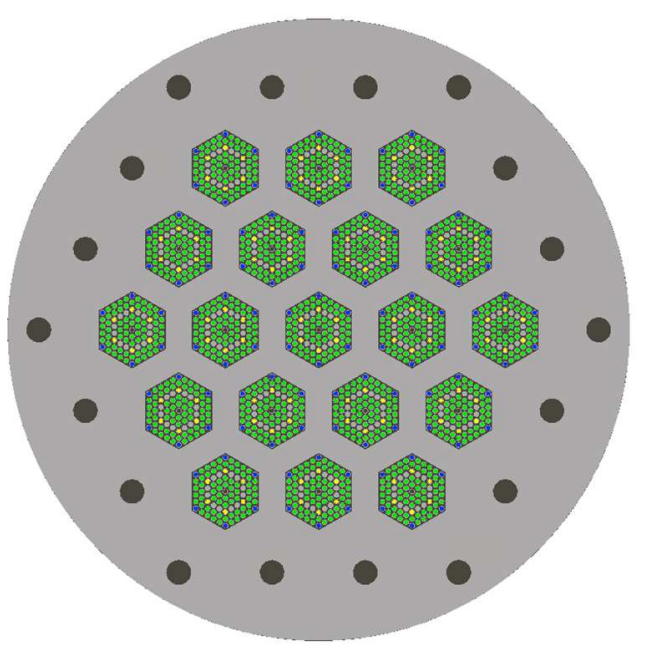

Figure 2. Full Core SmAHTR Model.

\subsection{TRISO Fuel}

The 66 fuel pins in the modified assembly are $1.1 \mathrm{~cm}$ radius, TRISO packed graphite rods with $0.3 \mathrm{~cm}$ thick graphite cladding. The TRISO used in the ORNL preconceptual design was modeled after a combination of UCO and $\mathrm{UO}_{2}$ TRISO fuel detailed in "AGR-2 Irradiation Test Final As-Run Report" [3]. The $\mathrm{UC}_{0.5} \mathrm{O}_{1.5}$ kernels with $19.75 \%$ enriched uranium are coated in several layers, as depicted in Figure 3 . While the preconceptual design does not explicitly state the composition of the TRISO, the core loading of 1,556.4 $\mathrm{kg}$ uranium $\left(19.75 \mathrm{wt} \%{ }^{235} \mathrm{U}\right)$ was verified with the following AGR-2 fuel properties in Figure 3 and $50 \%$ volumetric packing fraction given in the design paper. Note that the enrichment of the UCO kernel is explicitly for the SmAHTR preconceptual design, but it is not the enrichment listed for the AGR-2 experiment.

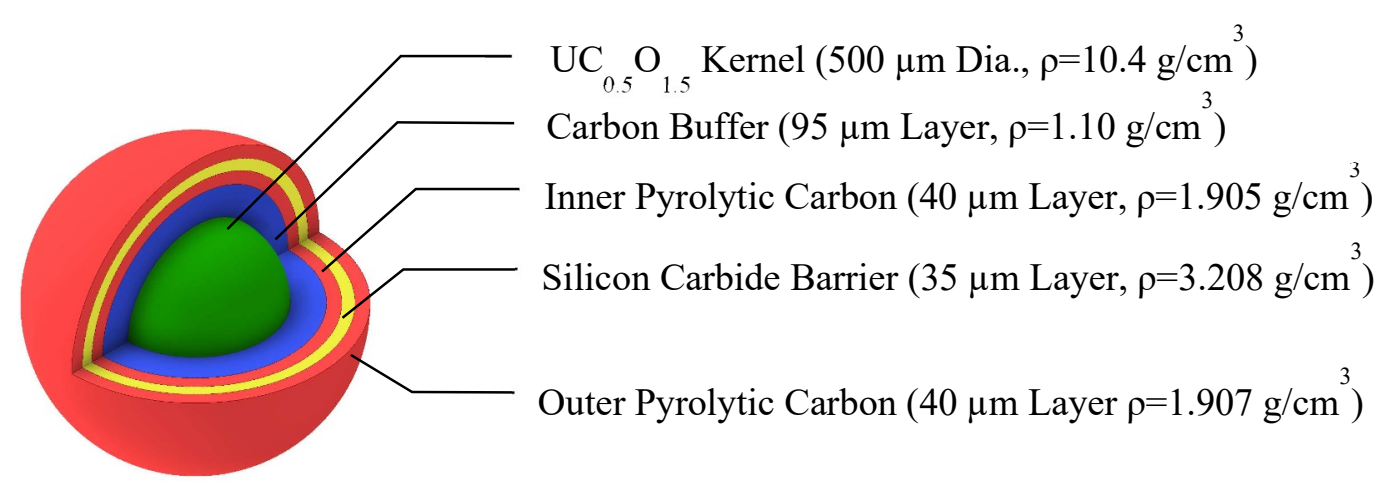

Figure 3 . TRISO Composition.

The high packing fraction for the TRISO is achieved by centering the particles in a layered, hexagonal lattice arrangement. The dimensions of the hexagonal lattice are a $0.0476 \mathrm{~cm}$ apothem and a $0.09417 \mathrm{~cm}$ height. This lattice arrangement also retains the physicality of the design by only centering a TRISO particle in lattice locations that do not allow pin boundaries to cut through particles. Specular reflective boundary 
conditions are established at the top and bottom planes of the assembly, thus providing an effectively infinite fuel pin that maintains heterogeneity of each particle. The kernels are suspended in graphite rods with properties listed in Table I at the end of section 2.5.

\subsection{Reactivity Control Mechanisms}

The SmAHTR pin fuel design is built with significant excess reactivity so that the core life can reach approximately four years, but to provide a complete 2D benchmarking set, control mechanisms were necessary to provide critical and subcritical configurations. The preconceptual design discussed potential materials for the control rods and burnable poison content, however, it did not definitively choose either locations in the assembly or material composition for them. Because boron is mentioned as the control material, and the pin design is similar to that in the Very High Temperature Reactor (VHTR) [4], some reactivity mechanisms are chosen using that design as reference.

\subsubsection{Control Rods}

For adjustable reactivity control, six control rods composed of homogeneous boron-carbide were specified in the six corners of the middle row of each assembly, which were originally pure graphite as seen in Figure 1. A seventh control rod location is specified in the center pin of the assembly, but it is left uninserted in all cases. The addition of the control rods is important to the benchmark problem, but note that the positioning of the control rods is not optimized. The boron-carbide mixture has a 55:1 C to $\mathrm{B}_{4} \mathrm{C}$ ratio, with a natural boron isotopic composition $\left(19.90 \%{ }^{10} \mathrm{~B}\right)$. Further information on the material composition of these rods is given in Table I below. The geometry of the control rod pins was modified slightly to meet two design features. First, a $3 \mathrm{~mm}$ thick graphite tube with an outer radius of $1.4 \mathrm{~cm}$ was specified for control rod guide tubes. This design choice maintains the same spacing between all pins and reduces the bypass flow in the uncontrolled reactor case [2]. Second, a clearance of $1 \mathrm{~mm}$ between the guide tube and control rod is specified to provide clearance for the inserted control rod. When the controlled case is considered, the six $1.0 \mathrm{~cm}$ control rods are in the corners of the middle row, and in the uncontrolled case, the $3 \mathrm{~mm}$ thick guide tube remains with FLiBe filling the space where the control rods would be otherwise.

\subsubsection{Burnable Poison}

The preconceptual design posed various methods for reactivity control, so suggestions from the paper as well as similarities between this pin type design and the Very High Temperature Reactor (VHTR) design (e.g., TRISO, pin type fuel, and graphite matrix) drove the decision to implement coated particles containing boron for burnable poison (BP) in the stylized SmAHTR problem set. The BP particles are embedded in graphite pins in each corner of the hexagonal assembly, previously dedicated as fuel pins, thus reducing the core loading. The particles are 200 micron diameter boron carbide kernels coated with an 18 micron thick carbon buffer layer and a 23 micron thick pyrolytic carbon outer layer [4]. They are packed at a 4\% volumetric packing fraction in the graphite rods within a rectangular lattice structure with side lengths of $0.063175 \mathrm{~cm}$ and a height of $0.07205 \mathrm{~cm}$ that maintain physicality by only filling lattice positions that will not cut off any fragment of the boron particles. The lattice differs from cubic by increasing spacing between the layers of particles/height of the lattice minimally to adjust the packing fraction of the particles without cutting them. The boron carbide within the particles contain ${ }^{10} \mathrm{~B}$ and ${ }^{11} \mathrm{~B}$ at a natural isotopic abundance: $19.9 \%$ and $80.1 \%$, respectively. These pins are the same size as each fuel pin $(1.1 \mathrm{~cm}$ radius $)$ with a $0.3 \mathrm{~cm}$ thick graphite cladding that maintains the same gap space between pins. Further specifications on the material composition are given in Table I below.

\subsection{Molten Salt Coolant}


The reactor is cooled by molten fluoride salt, $\mathrm{FLiBe}$ in a $2: 1$ ratio of ${ }^{7} \mathrm{LiF}$ to $\mathrm{BeF}_{2}$ enriched to $99.995 \%{ }^{7} \mathrm{Li}$. In the assembly, the FLiBe flows between the pins and within the control rod guide tube gap. There is also area in the graphite reflector coolant channels where FLiBe flows. The FLiBe material properties are given below in Table I.

\subsection{D Problem Setup}

Compression of the SmAHTR design into two dimensions requires appropriate consideration for heterogeneities in the axial direction. The compression of the system removes the natural boundaries of the reactor and implements specular reflective boundary conditions in its place. These reflective planes are specified at $\pm 15.0191 \mathrm{~cm}$ from the midpoint of the 4 meter tall reactor core, and are specified in such a manner to preserve physicality and heterogeneity of the TRISO and burnable poison particles by not cutting particles of either type. In addition to losing the full height of the core, the stylized problem loses the layer of FLiBe above and below the core. Note that in the 2D critical case, there is no partial insertion of control rods, so in the critical case for this reactor system, the control rods of an assembly are either fully inserted or fully removed from the core. Below, Table I provides specifications for the materials in the problem. Note that all materials pull from the ENDF/B-VII.0 endf70a library and endf70sab $S(\alpha, \beta)$ library if applicable.

Table I. Material Specifications.

\begin{tabular}{|c|c|c|c|}
\hline Components & $\begin{array}{l}\text { ZAID Material } \\
\text { Composition }\end{array}$ & Density (g/cc) & $\operatorname{Temp}(K)$ \\
\hline Control Rod - Boron Carbide & $\begin{array}{l}50100.199 \\
50110.801 \\
600014.00 \\
\end{array}$ & 2.266 & 800 \\
\hline $\begin{array}{l}\text { BP, Fuel, Moderator - Graphite Matrix } \\
\text { Control Rod - Graphite Guide Tube }\end{array}$ & 0060001.000 & 2.266 & 800 \\
\hline All Pins - Graphite Cladding & 0060001.000 & 2.266 & 800 \\
\hline BP - Boron Kernel & $\begin{array}{l}50100.199 \\
50110.801 \\
60000.250 \\
\end{array}$ & 2.47 & 1200 \\
\hline BP - Carbon Buffer & 0060001.0 & 1.00 & 1200 \\
\hline $\mathrm{BP}$ - Pyrolytic Carbon (PyC) Layer & 0060001.0 & 1.87 & 1200 \\
\hline Coolant - FLiBe & $\begin{array}{l}30060.0001 \\
30071.9999 \\
40091.0000 \\
90194.0000 \\
\end{array}$ & 1.94 & 900 \\
\hline TRISO - UCO & $\begin{array}{ll}092235 & 0.06650 \\
092238 & 0.26683 \\
006000 & 0.33333 \\
008016 & 0.33333\end{array}$ & 10.4 & 1200 \\
\hline TRISO - Carbon Buffer & 60001.0000 & 1.10 & 1200 \\
\hline TRISO - Inner PyC & 60001.0000 & 1.905 & 1200 \\
\hline TRISO - Outer PyC & 60001.0000 & 1.907 & 1200 \\
\hline TRISO - Silicon Carbide & $\begin{array}{cl}6000 & 0.50000 \\
14028 & 0.461485 \\
14029 & 0.023416 \\
14030 & 0.015436 \\
\end{array}$ & 3.208 & 1200 \\
\hline
\end{tabular}




\subsection{Radial Simplifications}

In the single assembly, the planar boundaries of the assembly are modeled to have specular reflective boundary conditions as the nature of single assembly modeling would suggest, but in the full core problem, the graphite reflector is modeled as the outer boundary of the problem. This simplification omits the FLiBe and reactor vessel surrounding the core, but it has a negligible impact on the neutronics of the problem.

\section{RESULTS}

\subsection{Solutions to the Single Assembly Problems}

In the single assembly benchmark problem set, the continuous energy Monte Carlo calculations were evaluated with MCNP 5, the $5^{\text {th }}$ release of the Monte Carlo N-Particle Transport Code [5], utilizing the ENDF/B-VII.0 data library. Specular reflective boundaries were specified around the graphite reflector and infinite extension of assembly in the axial direction. The pin fission density distributions were tallied in both cases, but could not be listed out due to page limitations, so the data is presented graphically in Figure 4 below. The two cases performed are the uncontrolled and controlled scenarios, where the controlled scenario has the six control rods inserted and the uncontrolled case has them all withdrawn. The resulting $\mathrm{k}_{\text {inf }}$ was $1.2117( \pm 15 \mathrm{pcm})$ for the uncontrolled case and $0.9987( \pm 15 \mathrm{pcm})$ in the controlled case. Additionally, the worth was calculated for the control rods and burnable poison rods in the assembly: 213 $( \pm 0.21) \mathrm{mk}$ and $337.4( \pm 0.20) \mathrm{mk}$, respectively. The relative pin fission density distribution is plotted in Figure 4 below.
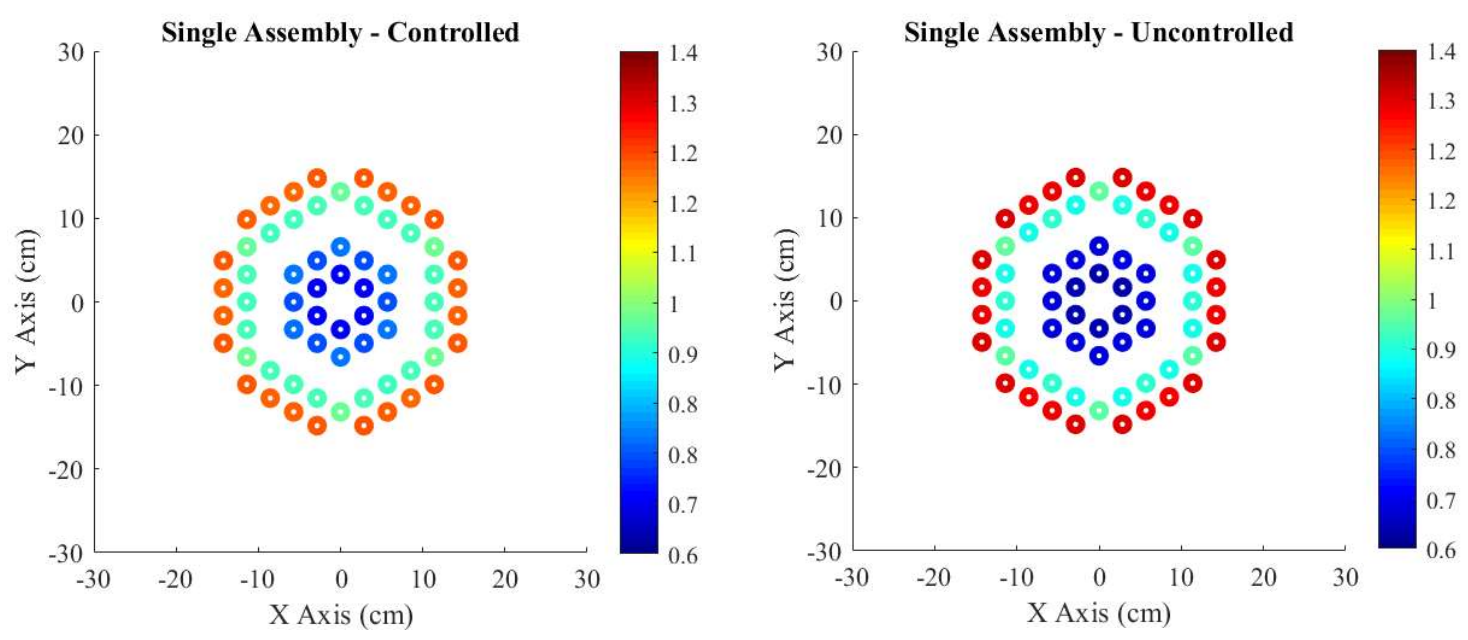

Figure 4. Relative Pin Fission Density Distribution in Single Assembly Cases

\subsection{Solutions to the Full Core Problems}

The full core problem set was evaluated in continuous energy with MCNP 5, the $5^{\text {th }}$ release of the Monte Carlo N-Particle Transport Code [5]. The pin fission density distributions were tallied in each core configuration. Three cases were evaluated for the full core: uncontrolled, controlled, and critical. Multiplication factors of $1.1436( \pm 12 \mathrm{pcm}), 0.9459( \pm 11 \mathrm{pcm})$, and $0.9909( \pm 12 \mathrm{pcm})$ for each case, respectively. The $\mathrm{k}_{\mathrm{eff}}$ of nearly one was achieved by taking the controlled core and withdrawing the control 
rods in every other outer-row assembly, as partial control rod insertion is not an option in 2D. Additionally, the worth was calculated for the control rods and burnable poison rods in the core: $197.6( \pm 0.16) \mathrm{mk}$ and $311.6( \pm 0.23) \mathrm{mk}$, respectively. Below in Figure 5, the relative pin fission density distribution is given for the controlled and uncontrolled full core cases. Since the core features $1 / 6^{\text {th }}$ symmetry, the plots are reduced to just those $1 / 6^{\text {th }}$ segments, yet each core configuration was simulated with the full core geometry. Figure 6 features the relative pin fission density distribution for the critical configuration case.
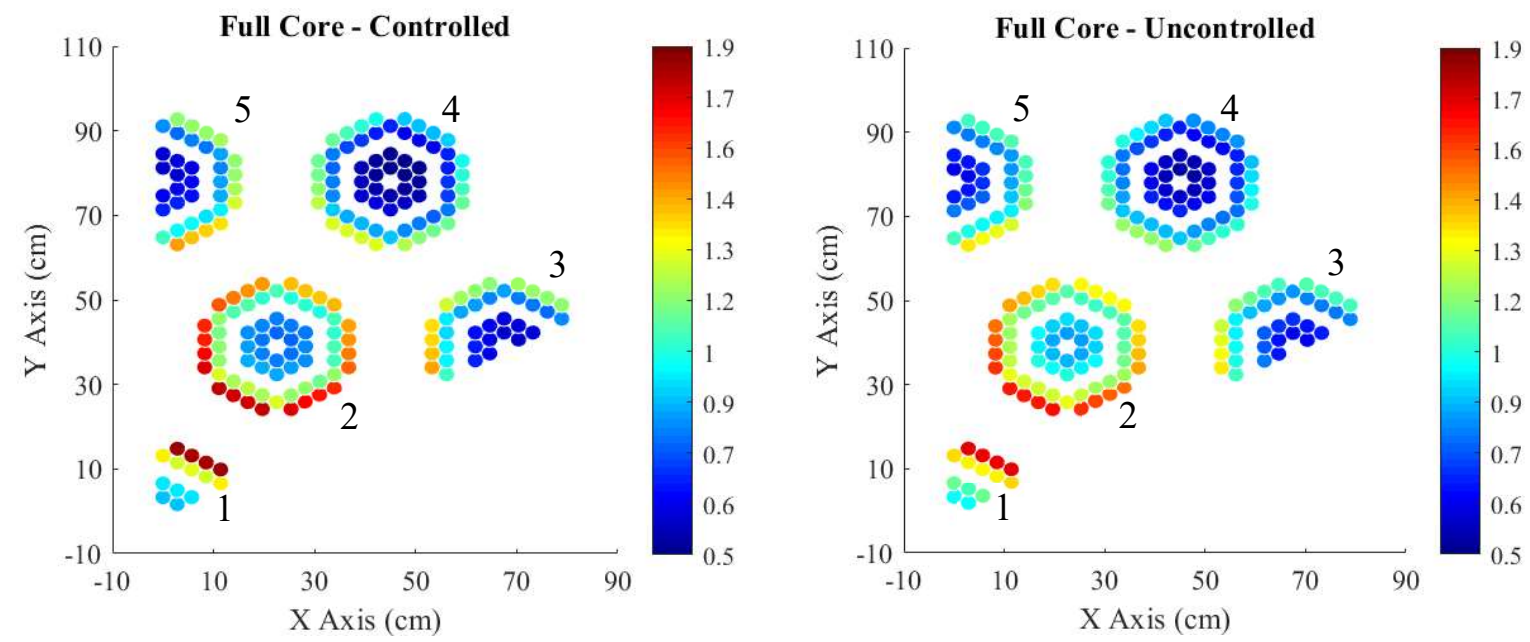

Figure 5. Relative Pin Fission Density Distribution in Full Core Controlled and Uncontrolled Cases

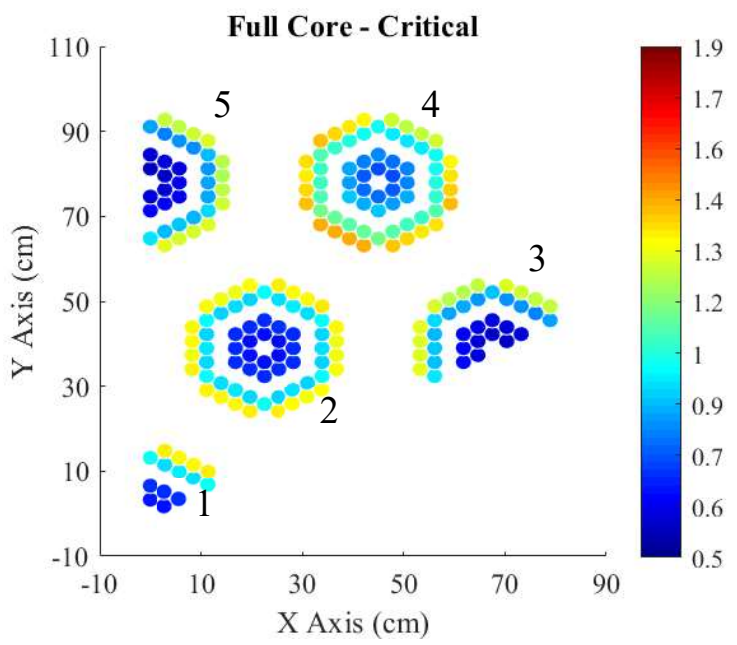

Figure 6. Relative Pin Fission Density Distribution in Critical Full Core Configuration

In each of the three cases, the relative assembly fission density was also calculated. Table II below shows the relative assembly-averaged fission density distribution in the fuel. These values are relative to the assemblies within the individual core configuration. Thus, Table II shows the flattening of the flux with near-critical configuration and the slight change in flux distribution with the controlled core compared to the uncontrolled.

Table II. Assembly Averaged Fission Density Distribution 


\begin{tabular}{|c|c|c|c|}
\hline Lattice & Uncontrolled Core & Controlled Core & Near-Critical Core \\
\hline 1 & 1.389 & 1.367 & 1.005 \\
\hline 2 & 1.212 & 1.190 & 0.998 \\
\hline 3 & 0.927 & 0.925 & 0.926 \\
\hline 4 & 0.801 & 0.798 & 1.087 \\
\hline 5 & 0.927 & 0.930 & 0.923 \\
\hline
\end{tabular}

\section{SUMMARY}

A set of 2D SmAHTR benchmark problems are developed and described in this paper. The benchmark set includes controlled and uncontrolled cases for the single assembly and full core in addition to a full core critical case. The problem is derived by simplifying the preconceptual SmAHTR design while retaining the neutronics characteristics such as multiple heterogeneities, but also filling gaps in reactivity control mechanisms. Continuous energy Monte Carlo solutions are also provided. It is anticipated that the benchmark problems and their reference solutions will be valuable to test the performance of neutron transport methods as well as its low order methods.

\section{ACKNOWLEDGMENTS}

This material is based upon work supported under an Integrated University Program Graduate Fellowship.

\section{REFERENCES}

1. S. Greene, et al., "Pre-Conceptual Design of a Fluoride-Salt-Cooled Small Modular Advanced High Temperature Reactor (SmAHTR)," United States: N. p., 2011. Web. doi:10.2172/1008830.

2. S. Chandrasekaran, "A Whole-Core, Steady-State Thermal Hydraulic Model for Cylindrical Pin Fuel Type Fluoride Salt-Cooled Small Modular Advanced High Temperature Reactor (SmAHTR)." Nuclear Technology. Accepted for Publication.

3. B. Collin, "AGR-2 Irradiation Test Final As-Run Report, Rev 2." United States: N. p., 2014. Web. doi:10.2172/1167549.

4. K. Connolly, et al., "Prismatic VHTR Neutronic Benchmark Problems," Nuclear Engineering and Design, vol. 285, 15 Apr. 2015, pp. 207-240., doi:10.1016/j.nucengdes.2014.11.044

5. X-5 Monte Carlo Team, i "MCNP - Version 5, Vol. I: Overview and Theory," LA-UR-03-1987 (2003). 